\title{
EDITORIAL
}

\section{Neonatal Jaundice: Continuing Concern and Need for Research}

$\mathrm{T}^{\prime}$ his issue of Pediatric Research contains an article with the provocative title "Are Moderate Degrees of Hyperbilirubinemia in Healthy Term Infants Really Safe for the Brain." This is an extremely important matter, one that has been considered and studied for many years. Recommendations and guidelines for the management of hyperbilirubinemia have been developed to avoid damage to the infant. One assumption has been that there is a "safe" level of plasma bilirubin, below which there is no risk of brain damage; alternatively it has been suggested that even "safe" levels may produce subtle neuronal damage.

This issue also includes several Commentaries and one Editorial, discussing this problem. The titles of the Commentaries - "Just when you thought it was safe," "Do we need to change what we are doing," "Does hyperbilirubinemia cause brain damage in apparently healthy newborns?"- suggest that there is considerable concern about this matter:

We have brought this matter to the attention of our readers because of the importance of the topic, the number of children who might be affected and the need for more caution in the management of the jaundiced newborn. The uncertainty and concern expressed in all these articles suggest the need for additional, carefully designed prospective studies to determine whether newborn babies are being harmed by what have been considered to be "safe" levels of plasma bilirubin. We welcome comments from our readers about this most important and unresolved problem.

Alvin Zipursky Editor-in-Chief

The optimal management of hyperbilirubinemia in healthy term infants remains unknown $(1,2)$. "Few issues in neonatal medicine have generated such long-standing controversy as the possible adverse consequences of neonatal jaundice and when to begin treatment. Questions regarding potentially detrimental neurologic effects from elevated serum bilirubin levels prompt continuing concern and debate, particularly with regard to the management of the otherwise healthy term neonate without risk factors for hemolysis" (2). The common goal expressed in the guidelines by the Committee on Fetus and Newborn, American Academy of Pediatrics (2) and the Fetus and Newborn Committee of the Canadian Paediatric Society (1) is to avoid plasma bilirubin concentrations that may results in kernicterus.

The apparent increase in the incidence of kernicterus among "healthy term infants" in North America (3) and Europe (4) may be due to a number of factors including better ascertaining and reporting of cases, or that the current guidelines are not followed or are not appropriate.

The case control study by Soorani-Lunsing et al. (5) and the commentaries published in the current issue of the journal should be seen as an incentive for continued research into hyperbilirubinemia of the healthy term infant. The authors found that moderate hyperbilirubinemia was associated with a significant increase in minor neurological dysfunction throughout the first year of life. They conclude that total serum bilirubin levels of $335 \mu \mathrm{mol} / \mathrm{L}$ should be avoided.

In the current report 20 cases with a plasma bilirubin level $>220 \mu \mathrm{mol} / \mathrm{L}$ were collected over an 8 months study period. The authors do not mention if these were consecutive cases one would have expected many more cases in an 8 months study period. These cases were likely selected from a larger group of patients, who would have met study inclusion criteria. One would also have expected more eligible controls during the study period. We do therefore not know how either the cases or the controls were selected from the larger group of potentially eligible patients. In case control studies usually at least 2-3 controls are selected for each case. The authors used a 1:1 ratio. There was no preset sample size calculation. Because of the small sample-size the $95 \%$ confidence interval around the point estimate of the outcome of developing minor neurological dysfunction at 12 months is very wide [OR 9.47 $(95 \% \mathrm{CI}=1-67 ; 53.65)]$. Plasma bilirubin levels were not measured in the control group. Thus plasma bilirubin or hyperbilirubinemia could not be entered as a variable in the logistic regression model. Only group status of having visible jaundices or not could be entered. As clinicians we are not very astute at estimating the total plasma bilirubin level by visual inspection of the baby. It is exceptional in a case control study that the key variable under study, in this case total plasma bilirubin, was not measured in both cases and control. A dose response could therefore only be studied among the cases.

The cesarean section rates differed among cases and controls; maternal education also differed between the two groups (possibly because of the small sample size this did not reach statistical significance). The authors provide only few base-line characteristics of the cases and controls.

Four babies in the study group and 2 in the control group were born preterm (36 wk). The practice parameters by the American Academy of Pediatrics and the Canadian Paediatric Society address hyperbilirubinemia only in term infants (37 wk or more) $(1,2)$. Preterm infants should have been excluded 
from the current study if the objective of the study was to overturn the current recommendations.

I am not certain that the assessment tools used at follow-up have been validated. The early assessments were not performed blinded to group allocation. In the newborn period there was a wide age range among the infants at the time of assessment.

The question asked by the authors: "Are moderate degrees of hyperbilirubinemia in healthy term neonates really safe for the brain?" is an important one, which needs an urgent answer. In my opinion and because of the serious methodological flaws in the current study the authors have not provided an unbiased answer to their question. This can only be achieved by conducting a well-designed cohort study. Such a study should have an appropriate sample size, measure plasma bilirubin levels in all enrolled infants and use validated assessment tools for the long-term (at least 18 months of age) outcomes. Individuals blinded to the neonatal conditions should undertake the assessments of neuro-motor function at 18 months.

Arne Ohlsson
Editor

\section{REFERENCES}

1. Fetus and Newborn Committee Canadian Paediatric Society, College of Family Physicians of Canada 1999 Approach to the management of hyperbilirubinemia in term newborn infants. Pediatr Child Health 4:161-164.

2. American Academy of Pediatrics 1994 Management of hyperbilirubinemia in the healthy term newborn. Pediatrics 94:558-565.

3. Johnson L, Brown AK 1999 A pilot registry for acute and chronic kernicterus in term and near-term infants. Pediatrics 104 (suppl):736(abstr)

4. Ebbesen F 2000 Recurrence of kernicterus in term and near-term infants in Denmark. Acta Paediatr 89:1213-1217.

5. Soorani-Lunsing I, Woltil HA, Hadders-Algra M 2001 Are moderate degrees of hyperbilirubinemia in healthy term neonates really safe for the brain? Pediatr Res 2001;50:701-705 